\title{
Zweckscheinbarkeit Über eine Kategorie im Umfeld von Kant und Schiller
}

Wenn man über Unnützes Wissen in Bezug auf ästhetische Bildung nachdenkt, ist es nur eine Frage der Zeit, bis man auf Schillers Briefe Über die ästhetische Erziehung des Menschen (1795) stößt. Schiller formuliert dort eine Kulturkritik $^{1}$ für das späte 18 . Jahrhundert, die einmal besagt, dass sein Zeitalter sämtliche Bereiche des Lebens nur in Bezug auf einen ökonomischen Nutzen denken kann, was die menschliche Existenz vereinseitigt und unglücklich macht, und das andere Mal, dass theoretische Großprojekte wie die Aufklärung und die Französische Revolution nicht realisiert werden können, weil sie den Menschen - auch wieder einseitig - nämlich nur über die Vernunft erreichen. Dies hat zur Folge, dass die Ideen der Aufklärung zwar gedacht und unterrichtet, aber nicht oder nur schlecht umgesetzt werden können, da sie nicht der Realität des menschlichen Handelns entsprechen. Deshalb bringt Schiller eine Form des Unnützen Wissens ins Spiel, das er in der Kunst findet. Da die Aufklärung - wie z.B. mit dem kategorischen Imperativ Kants - sich nur an die Vernunft richtet, wird ergänzend eine Form des ästhetischen Wissens eingebracht, welches auch die Sinne ansprechen soll.

In Schillers Terminologie der Ästhetischen Briefe übersetzt, drückt sich dieser Grundgedanke der ästhetischen Bildung so aus: Die Dominanz rationaler Kopfgeburten ist das Resultat eines zu übermächtig gewordenen »Formtriebs«. Der rationale »Formtrieb $\aleph^{2}$ ist zwar nützlich, weil er der ungeformten

1 Das Verständnis von Schiller als einen frühen Kulturkritiker übernehme ich von Georg Bollenbeck, Von der Universalgeschichte zur Kulturkritik, in: Lothar Ehrlich/Georg Bollenbeck (Hg.), Friedrich Schiller. Der unterschätzte Theoretiker, Wien/Köln/Weimar 2007, S. 11-26; sowie Georg Bollenbeck, Eine Geschichte der Kulturkritik. Von Rousseau bis Günther Anders, München 2007, S. 76-111. Bollenbeck betrachtet Schiller, neben Rousseau, als den ersten Kulturkritiker, da er über eine kulturkritische Triade verfügt, die ihr Zeitalter im Verfall sieht, diese Kritik durch den rückblickenden Vergleich mit einer idealisierten - meist griechischen - Vergangenheit begründet, und, unter der Bedingung, dass sich die Gegenwart ein Beispiel an dieser Vergangenheit nimmt, über einen ebenso idealisierten Ausblick in eine mögliche Zukunft verfügt. In den Ästhetischen Briefen Schillers findet sich diese Kulturkritik vor allem in den ersten Briefen 2-6.

2 Friedrich Schiller, Über die ästhetische Erziehung des Menschen, in: Ders., Theoretische Schriften, Bd. 8, hg. von Rolf-Peter Janz, Frankfurt a.M. 1992, 12. Brief, S. 602. 
Existenz Form (also Struktur, Dauer, Mäßigung und Idee) verleiht, jedoch nimmt er im Laufe des menschlichen Entwicklungsprozesses überhand und dominiert den anderen menschlichen Grundtrieb, den "Stofftrieb «. ${ }^{3}$ Letzterer befindet sich ursprünglich in einem ausgeglichenen Verhältnis mit dem Formtrieb, den dieser als bloßer Stoff (ungeformte Materie, Sinnlichkeit und Irrationalität) zu seiner Formgebung durchaus benötigt. Nimmt aber die Form überhand, erstickt das Sinnliche und das Resultat sind die oben beschriebenen unglücklichen Versuche, Ideen aus dem Bereich der reinen Vernunft zu realisieren. Zur Lösung dieses Problems führt Schiller nun die Kunst in Form einer ästhetischen Erziehung an: Wenn Form- und Stofftrieb unausgeglichen sind, dann müssen sie ausgeglichen werden. Da dies aber nicht von selbst geschieht, bedarf es einer vermittelnden Instanz, und dies leistet die Kunst. Sie bringt Form und Stoff in das gewünschte Gleichgewicht, für das Schiller den Begriff "Spieltrieb « ${ }^{4}$ prägt. Mittels des Spieltriebs vermag der Mensch nun seine Ideale zu realisieren, da er ihre Umsetzung nicht mehr einseitig von der Vernunft gesteuert zu vollbringen versucht. Während man sich nach Kant dazu überwinden muss, den kategorischen Imperativ umzusetzen, wird die Idee des Guten mit Hilfe der Kunst gewissermaßen in einen Trieb verwandelt, so dass sich das Gute automatisch, wie andere Triebe auch, von selbst realisiert. Diesen Weg der Verwirklichung der Ziele der Aufklärung über die Kunst nennt Schiller den "ästhetischen Umweg ${ }^{5}$, da der Verstand seine Pläne nicht mehr direkt umsetzen darf, sondern die Kunst zu Hilfe nehmen muss.

Auf diesem Umweg vollzieht sich auch das, was wir im Folgenden als das Ästhetisch-Zweckfreie in Bezug auf einen Nutzen untersuchen möchten. Die von Schiller ins Spiel gebrachte Kunst kann, da sie sich gegen die Welt der Zwecke richtet, durchaus als sunnütz` verstanden werden, denn die ästhetische Enklave des Bildungsprozesses konstituiert sich über Muße, Eigensinn und Ungeplantheit, was in Bezug auf die Arbeitswelt durchaus als nutzlos verstanden werden kann, es dann aber trotz allem auch wieder nicht ist, weil sie ja etwas hervorbringen soll, nämlich Bildung. Deshalb verfolgt das Unnütze eine Aufgabe - und diesen Nutzen, der vom Unnützen hervorgerufen und geprägt wird, möchten wir genauer untersuchen. Da wir uns mit diesem Changieren zwischen Nutzlosigkeit und Nutzen auf einer nur schwer zu definierenden Schwelle ansiedeln, soll im Folgenden der Begriff

\footnotetext{
Ebd.

Friedrich Schiller, Über die ästhetische Erziehung des Menschen, 14. Brief, S. 607.

5 Ebd., 15. Brief, S. 614.
} 
ZZweckscheinbarkeit ${ }^{6}$ Klarheit verschaffen. Er führt zu einer widersprüchlichen Verbindung zwischen Schönheit und Bildung bzw. zwischen Nutzen und Nutzlosigkeit, und die Grundfrage lautet: Wie kann etwas, das von jeder zweckhaften Bestimmung frei ist, dennoch einen Zweck entwickeln, nämlich den, die ästhetische Erziehung des Menschen zu gestalten? Wie operiert dieser besondere Zweck des Zweckfreien?

\section{Schiller: Zweckscheinbarkeit}

Schillers Ästhetische Briefe postulieren für den Menschen zunächst jedoch einen Zustand, der wenig Nutzen hervorzubringen verspricht: Der Spieltrieb soll den einseitig bestimmten Menschen in einen 'Nullzustand k versetzen, in dem er noch einmal die Möglichkeit erhält, snull bestimmt<, d.h. von den Folgen der Moderne undeterminiert, das Verhältnis zwischen Form- und Stofftrieb neu auszutarieren. Die historische Entwicklung des Nullzustands skizziert Schiller dementsprechend utopisch: Am Beginn seiner Geschichte ist der Mensch dem Zwang ausgesetzt, eine schlechte Einheit mit der Natur eingehen zu müssen. Er unterliegt zunächst passiv ihrer Gewalt und muss mit ihr "Eins " ${ }^{7}$ sein. Diese problematische Einheit wird noch intensiviert, indem er ihrer Anforderung nachkommt, sich in der Welt zweckhaft zu betätigen, damit er sein Überleben sichern kann. Von Freiheit kann noch keine Rede sein, da er mit seinen Handlungen nur auf die bedrohenden Einflüsse seitens der Natur reagiert. Um diese allein dem Zweck des Überlebens untergeordnete Lebensweise in eine freiheitliche umwandeln zu können, sucht Schiller nach einer Möglichkeit, das Verhältnis des Subjekts zur Welt auf "Null $\aleph^{8}$ herunterzufahren, so dass es eine Distanz zwischen ihm und der Welt gibt, in der er seine Autonomie ausbauen kann. Diese menschliche Eigengesetzlichkeit begründet sich für Schiller in einem ästhetischen Verhältnis zur Welt, da der Mensch mit dieser Zweckfreiheit (von der Natur) seinen eigenen Bedürfnissen nachgehen kann, die in dem Sinne zweckfrei sind, dass sie ihm erlauben, sich frei zu machen von den Anforderungen, welche

$6 \quad$ Zu einer ausführlichen Diskussion der Zweckscheinbarkeit vgl. meine Dissertation, Das Labyrinth der ästhetischen Einsamkeit. Eine kleine Theorie der Bildung, Würzburg 2015.

7 Friedrich Schiller, Über die ästhetische Erziehung des Menschen, 25. Brief, S. 655.

8 Ebd., 21. Brief, S. 636. 
die Natur an ihn stellt. In der Realisierung dieser Unabhängigkeit begründet sich »das erste liberale Verhältnis des Menschen zu dem Weltall«. ${ }^{9}$

Da dieser Annullierung der Wunsch nach Minderung des Zweckhaften zugrunde liegt, diese aber dennoch ein Anliegen verfolgt, beschreibt sie exemplarisch den Widerspruch des Zweckfreien: Einerseits zeichnet dieser Nullzustand sich dadurch aus, dass er das Subjekt unbestimmt lässt, andererseits bereitet er das Subjekt auf etwas vor, welches es wieder neu - und besser als vorher - bestimmt. Der Nullzustand wird im Rahmen des ausgleichenden Stofftriebs eingeführt und dient als Kristallisationspunkt der Nullstelle, die in der Balance zwischen Form- und Stofftrieb entsteht. Seine Ausprägung erhält der Nullzustand anschließend in der Form des Spieltriebs im ästhetischen Zustand. Und genau hier setzt unsere Frage ein. Wenn das Zweckfreie zugleich annullieren und formen kann, wie lässt sich das darstellen, wie darüber sprechen, was geht in diesem ungreifbaren Zustand vor, von dem es heißt: "In dem ästhetischen Zustand ist der Mensch also Null, insofern man auf ein einzelnes Resultat, nicht auf das ganze Vermögen achtet, und den Mangel jeder besonderen Determination in ihm in Betrachtung zieht «? ${ }^{10}$ Ist der Mensch ästhetisch geworden, dann ist er aus der Sicht des Verstandes nichts oder eben "Null« und kann kein »einzelnes Resultat« hervorbringen und nicht einer »besonderen Determination" entsprechen, weil es ihn nicht zum Leben befähigt. Die Schönheit, so schreibt Schiller nur einige Zeilen weiter, "findet [nämlich] keine einzige Wahrheit, hilft uns keine einzige Pflicht erfüllen und ist, mit einem Worte, gleich ungeschickt, den Charakter zu gründen und den Kopf aufzuklären«. ${ }^{11}$ Jedoch verfügt die Schönheit über einen individuellen Wert, insofern sie den Menschen nicht dazu drängt, alles sogleich auf etwas Allgemeines zu beziehen und auf die Realität zu übertragen. In dieser ästhetischen Bestimmungslosigkeit zeigt sich dann, dass sein allgemeines "Vermögen« als Mensch keineswegs annulliert wurde, sondern dass es ihm durch die Abwesenheit der Verstandeskontrolle "nunmehr von Natur wegen möglich gemacht ist, aus sich selbst zu machen, was er will«. ${ }^{12}$ Diese Möglichkeit innerhalb des Nullzustandes gibt ihm seine Würde zurück. "Null« ist der Mensch also nur als Verstandeswesen, das im ästhetischen Zustand keinen Begriff und somit auch kein Resultat zu erfassen vermag und deshalb eine »leere Unendlichkeit « ${ }^{13}$ vor

9 Ebd., 25. Brief, S. 655.

10 Ebd., 21. Brief, S. 636.

11 Ebd.

12 Ebd.

13 Ebd. 
sich hat. Derselbe ästhetische Nullzustand erscheint jedoch als eine »erfüllte Unendlichkeit «, ${ }^{14}$ wenn man sich in diesem annullierten Stadium auf sein individuelles und ästhetisches Vermögen als Mensch konzentriert, das ihm in der Möglichkeitsform ein Dasein verspricht, das seiner Würde und nicht der Pflicht des Verstandes gehorcht, ihn realitätsgerecht zu determinieren. Anders gesagt: Der Nullzustand ermöglicht dem Menschen das Recht auf Kunst, das ihm aber nur in Abgrenzung zum Leben ermöglicht werden kann.

Für die Beschreibung dieses annullierten oder zweckfreien Feldes führen wir den Begriff 'Zweckscheinbarkeit`ein, der uns als Schlüsselbegriff zum Verständnis des Zweckfreien dienen wird. Mit ihm lässt sich eine Beschreibung des Zweckfreien skizzieren, die verdeutlicht, dass der in dieser Beziehung entstehende ästhetische Nullzustand nicht zweck- oder nutzlos ist. Das Zweckfreie ereignet sich in unserem Verständnis der ästhetischen Bildung nämlich nur scheinbar: Der Schein ist zwar losgelöst von der Sphäre der eindeutigen Zwecke, was ihn zunächst auch zweckfrei aussehen lässt. Doch Zwecke sind auch hier gegenwärtig, aber nur für eine andere Sphäre bestimmt, in die sie gelangen, wenn sie ihre unmittelbare Zweckhaftigkeit ablegen. Zwecke werden zunächst scheinbar, damit sie sich der Realität entziehen können. Da die ersten zweckfreien Momente eine zeitliche Dauer brauchen, um sich auszubauen und bildend wirken zu können, müssen diese Momente Beständigkeit haben. Das passiert durch die Umwertung der Zwecke im ästhetischen Schein, der die Zwecke scheinbar wirken lässt, um sie der Arbeitswelt zu entziehen und anschließend wiederum die Zwecklosigkeit scheinbar werden lässt, damit sie im Zweckfreien wie konventionelle Zwecke wirken kann. Ihre Zweckfreiheit besteht demnach darin, dass das Subjekt seine Zwecke selbst wählen kann. Zweckfreiheit operiert als ein Setzen von Zwecken in der Freiheit. Verdichten sich die scheinbaren Zwecke zu einem Prozess, formt sich eine ästhetische Gegenwelt aus.

Es zeigt sich, dass in der ästhetischen Welt das Zweckrationale veredelt ist und durch den ästhetischen Schein zu scheinbaren Zwecken wird, die einer schönen Welt würdig sind. Kunst hemmt die ständige Ausbreitung einer zweckhaften Weltorientierung und ersetzt sie - nicht durch völlig zweckfreies, sondern - durch zweckhaftes Denken, Planen und Handeln im Schein, wodurch die Unterschiede zwischen Zweck und Kunst aufgehoben werden. Diese Zwecke sind dem Subjekt nicht mehr entgegengesetzt, sondern ihre

14 Ebd. 
die Freiheit auswringenden zweckrationalen Eigenschaften werden in sicherem Abstand gehalten.

\section{Kant: Zweckscheinbarkeit versus Zweckmäßigkeit ohne Zweck}

$\mathrm{Zu}$ Beginn dieses Abschnitts muss zunächst hervorgehoben werden, dass der zentrale Begriff der vorliegenden Untersuchung - Zweckfreiheit -, in seiner Diskussion nie eine philosophische oder kulturtheoretische Definition erfahren hat. Zweckfreiheit versteht sich vielmehr als ein Sammelbegriff, der uns vor allem deshalb so sehr interessiert, weil er außerhalb des philosophischen Diskurses - im Feuilleton oder im Alltag - ein gebräuchlicher Ausdruck ist. Suchen wir ihn in der Süddeutschen oder Frankfurter Allgemeinen Zeitung, findet er sich regelmäßig im Kulturteil, und lassen wir das Internet die gesamte Welt danach durchkämmen, wird das Suchergebnis so umfangreich, dass sich Google zu einem endlos erscheinenden, ja an Schiller erinnernden `Nullzustand ausdehnt.

Die Durchsicht dieser Resultate ergibt, dass - unter Berufung auf Kant und Schiller - "Zweckfreiheit« oder "Zweckfrei« stets dann auftaucht, wenn von Erfahrungen die Rede ist, die alltägliche Zweckanforderungen kritisch gegenüberstehen und kompensieren möchten - und dementsprechend in einem gewissen Sinne snutzlos sind (z.B. musische Erziehung, WorkLife-Balance oder Theaterbesuche). Durch diesen simplen Gebrauch wird "Zweckfreiheit" etwas sehr Vages, auf das man sich immer berufen kann, ohne es wirklich verstanden haben zu müssen oder sich der Schwierigkeit des Verhältnisses zwischen dem Zweckfreien und dem Zweckhaften bewusst zu sein. Unsere Untersuchung widerspricht dieser Leichtigkeit und Unbestimmtheit des Zweckfreien.

Mit Zweckscheinbarkeit haben wir in Auseinandersetzung mit Schillers Ästhetischen Briefen einen Begriff eingeführt, der das Ambivalente des Zweckfreien besser zum Ausdruck bringt und sich nicht in vagen Vorstellungen verliert. Wir verstanden Zweckscheinbarkeit als einen Vorgang, der die Zwecke nicht abschafft, jedoch das Setzen von Zwecken in einer Sphäre erlaubt, die den Bedürfnissen des Subjekts nicht entgegengesetzt ist. Es kann dort mit den Zwecken spielen und zwar in dem Sinne, dass er die Zwecke verfolgen kann, ohne aber verpflichtet zu sein, sie auch tatsächlich im Leben zu aktivieren. Wir sagten auch, dass diese Art von spielerischer Zweckgerichtetheit dem Hervorbringen von Zwecken in der Freiheit gleichkommt, 
deren Aktivität jedoch nicht geringer ist als im zweckgerichteten Alltag. Der Hauptunterschied zwischen den Zwecken in der Realität und denen im ästhetischen Zustand wurde nämlich darin gesehen, dass die Zwecke erst im Prozess der Ausübung, d.h. im Nachhinein, verständlich werden und nicht bereits im Vorfeld feststehen müssen. Da die Zwecke demzufolge jederzeit auch zweckfrei sein und sich auflösen können, während das Zweckfreie jederzeit zu einem konkreten Zweck werden kann, ist dieser Moment ihrer Entstehung ein scheinbarer.

Im Folgenden wenden wir uns nun Kant zu, da er mit der Zweckmäfigkeit ohne Zweck eine Formel prägte, die das Resultat einer erstmaligen systematischen Ausarbeitung des Zweckfreien ist und noch heute die Grundlage der Diskussion eines jeden ästhetischen Weltverhältnisses prägt. Wir möchten nach der Aussagekraft der Kant'schen Formel fragen und zeigen, dass sich Zweckscheinbarkeit besser dazu eignet, dem Zweckfreien näherzukommen, weil es von scheinbaren Zwecken bzw. scheinbaren Freiheiten von Zwecken ausgeht und deshalb im Gegensatz zu Kant kein reines ästhetisches Urteil (also eines ohne jeglichen Zweck) anstrebt, sondern den prozessualen Charakter ästhetischer Momente betont, der eben nicht immer rein ästhetisch sein muss. Dadurch kann gezeigt werden, dass gerade Kants Wunsch nach dieser reinen Ästhetik der Ausgangspunkt für eine ästhetische Bildungsidee wurde, die das Zweckfreie so eigentümlich sprach- und bildlos gemacht hat. Wir werden Kants Zweckfreiheitskonzeptionen zunächst vorstellen, um das hier vorliegende Problem genauer zu beschreiben, dann aber die Richtung der Untersuchung verändern und das Problem außerhalb seiner Theorie und Begrifflichkeit (Zweckmäßigkeit, Zweckmäßigkeit ohne Zweck und seiner Definition der Schönheit) verfolgen.

Wie Kant am Beginn der Kritik der Urteilskraft schreibt, gründet sich das ästhetische Urteil auf ein Gefühl der Lust, das nicht primär auf den Verstand und sein begriffliches Instrumentarium für Erkenntnisurteile zurückgeht, sondern auf ein besonderes Verhältnis des Verstandes mit der Anschauung. ${ }^{15}$ Die Anschauung nimmt den schönen Gegenstand wahr, jedoch geht

15 Immanuel Kant, Kritik der Urteilskraft, hg. von Heiner F. Klemme, Hamburg 2009, AA § 1-9. Zur weiteren Diskussion der Begrifflichkeit im ästhetischen Urteils bei Kant vgl.: Paul Guyer, Kant and the Claims of Taste, Cambridge MA 1997, S. 85f.; Dieter Heinrich, Aesthetic Judgment and the Moral Image of the World, Stanford 1994, S. 40-52; Rudolf Makkreel, Imagination and Interpretation in Kant, Chicago 1990, S. 49-58, sowie David Bell, The Art of Judgment, in: Mind 96 (1987), S. 221-244; Paul Crowford, Kant's Aesthetic Theory, Madison 1974, S. 88f.; und Paul Crowther, The Kantian Sublime, Oxford 1989, S. 55f. 
sie keine Einheit mit einem Begriff ein. Durch dieses Zurückdrängen der Begriffe wird für Kant das Geschmacksurteil interesselos, was bedeutet, dass das Gefühl der Lust bei der Betrachtung eines schönen Gegenstands nicht mit dem Interesse an der materiellen Existenz dieses Objekts verbunden ist. Stattdessen entsteht lediglich ein Wohlgefallen, das Kant »in der bloßen Betrachtung «, ${ }^{16}$ also beim Verweilen dieser Anschauung ansiedelt. Damit wird das ästhetische Wahrnehmen vom Angenehmen abgegrenzt, dem Kant ein Interesse an einem realen Gegenstand unterstellt, und auch vom Guten, das aufgrund seiner Universalität auf einen Begriff zurückgeht, d.h., wo Verstand und Anschauung eine klar definierte Einheit bilden. Somit kann Kant einmal im Hinblick auf die Interesselosigkeit folgern: "Ge s chmack ist das Beurteilungsvermögen eines Gegenstands oder einer Vorstellungsart durch ein Wohlgefallen ohne alles Interesse. Der Gegenstand eines solchen Wohlgefallens heißt schön. " ${ }^{17}$ Und das andere Mal im Hinblick auf die Begriffslosigkeit: »Schön ist das, was ohne Begriff [...] gefällt. ${ }^{18}$ Die Unabhängigkeit von Verstand und Einbildungskraft führt zu einem »freien Spiel ${ }^{19}$ zwischen diesen beiden Polen, das dem Subjekt das Wohlgefallen am schönen Gegenstand verschafft. - Und genau hier steckt die größte Schwierigkeit der ästhetischen Theorie Kants, weil nicht eindeutig ist, was im Verlauf der Urteilsbildung mit den Begriffen geschieht, da sie, wenn der Verstand im Spiel ist, nicht einfach verschwinden können. Bevor wir uns jedoch der Diskussion dieses Problems zuwenden können, müssen wir mit Kants Gebot der Universalität des Geschmacksurteils und seiner Definition als »Zweckmäßigkeit ohne Zweck« noch zwei weitere Momente seiner Ästhetik einführen.

Ein weiterer Widerspruch des Geschmacksurteils geht ebenfalls auf die kurz angedeutete rätselhafte Begriffslosigkeit zurück. Ein Geschmacksurteil - wie ein begrifflich-logisches Urteil - wird universal gefällt, was für Kant bedeutet, dass ein Individuum sein subjektives Wohlgefallen im ästhetischen Urteil notwendig verallgemeinert und davon ausgeht, dass die anderen der gleichen Ansicht sind und den gleichen Gegenstand ebenfalls als schön empfinden müssen. Ein logisches Urteil, z.B. dass die Wiese grün ist, basiert auf einem Begriff und unterstellt damit als Urteil seine allgemeine Geltung. Die logische Überprüfbarkeit entfällt jedoch, wenn das Urteil ästhetisch ist, da es jetzt nicht mehr auf einen Begriff zurückgeht. Gefällt einem also die grüne

\footnotetext{
16 Ebd., S. AA 204.

17 Ebd., S. AA 211.

18 Ebd., S. AA 219.

19 Ebd., S. AA 217.
} 
Wiese im ästhetischen Urteil, so kann diese Aussage nicht weiter bewiesen werden. Dass dieses Gefallen aber dennoch den Anschein der Universalität besitzt - also scheinbare Begriffe produziert -, liegt an dem sich in einem Spielzustand befindenden Verstand, der irgendwie immer noch anwesend ist, aber der Anschauung keine Begriffe mehr aufdrängt. (Man könnte zum besseren Verständnis bildlich und vereinfacht auch sagen, dass er experimentelle Begriffe produziert, die probehalber formuliert werden, aber sich auch wieder auflösen können.) Der dritte Punkt des ästhetischen Urteils lautet deshalb für Kant: »S chön ist das, was ohne Begriff allgemein gefällt. ${ }^{20}$ Geht die subjektive Universalität auf einen scheinbar anwesenden Begriff zurück, so könnte man ebenfalls argumentieren, dass die berühmte Formel von einer "Zweckmäßigkeit ohne Zweck" im Geschmacksurteil auf scheinbare oder experimentelle Zweckvorstellungen zurückgeht, da sie aus der gleichen formalen Verstandestätigkeit des "freien Spiels ${ }^{21}$ der Erkenntniskräfte resultieren. Dazu werden wir uns im Folgenden Kants unterschiedliche Definitionen der Zweckmäßigkeit anschauen, um herauszufinden, welche Form der Zweckmäßigkeit aus seiner Naturteleologie auf die ästhetische Theorie angewandt wird und was in dieser Transformation mit der Begrifflichkeit passiert. Kants Verständnis der "Zweckmäßigkeit« stammt aus seiner Naturphilosophie des 2. Teils der Kritik der Urteilskraft, wo er der Frage nachgeht, ob der Natur eine teleologische Ordnung zugrunde liegt. Im $\mathrm{Zu}$ sammenhang mit dieser Problematik unterscheidet er dann zwei verschiedene Arten der Zweckmäßigkeit - die formal objektive der Vernunft und die real objektive des Verstandes. (I) Die formal objektive Zweckmäfigkeit ist weder subjektiv noch ästhetisch, sondern ein a priori operierendes Vermögen der Vernunft: Das vom Menschen in der Natur Wahrgenommene strukturiert die Vernunft in der Art, dass man ihr notwendigerweise eine sinnvolle Ordnung unterstellt, die zum jetzigen Zeitpunkt zwar noch nicht bekannt ist und auch in der Anschauung nicht zum Vorschein kommt, jedoch den Betrachter ideell davon überzeugt, dass alles mit allem auf einen bestimmten Endzweck hin organisiert ist: »Die Zweckmäßigkeit ist hier objektiv und intellektuell, [...], denn sie drückt die Angemessenheit der Figur zur Erzeugung vieler abgezweckten Gestalten aus und wird durch Vernunft erkannt. ${ }^{22}$ Die Zweckmäßigkeit liegt jedoch nicht in den Begriffen und Dingen, sondern allein in der apriorischen Ordnungsvorstellung der Vernunft. (II) Die real objektive

20 Ebd., S. AA 229 [Hervorh. MG].

21 Ebd., S. AA 217.

22 Ebd., S. AA 362. 
Zweckmäfigkeit dagegen gründet sich auf eine tatsächliche Anschauung, die der Verstand mit einem Begriff verbindet und damit reale Gegenstände in eine kausale Ordnung eingliedert. Diese Form der Zweckmäßigkeit ist real, da sie nicht auf eine Vorstellung, sondern auf Beobachtungen zurückgeht. Sie konzentriert sich auf »existierende Dinge [...], die empirisch gegeben sein müssen, um erkannt werden zu können, und nicht eine bloße nach einem Prinzip a priori bestimmte Vorstellung in mir. Daher die letztere (empirische) Zweckmäßigkeit als real von einem Begriffe eines Zwecks abhängig ist. ${ }^{23}$ Die mit der realen Zweckmäjsigkeit der Natur operierende Forschung dient demzufolge einem konkreten Zweck, während die Vernunft eine fiktive Ordnung in die Natur projiziert, um der Mannigfaltigkeit der Naturerscheinungen einen Leitfaden zu geben, der als regulative Idee die reale Forschung strukturiert und antreibt. Da die formale Zweckmä/sigkeit sich nur mit ideellen Endzwecken befasst, löst die Kant'sche Ästhetik den Betrachter aus der realen Zweckmäjsigkeit des Verstandes in dem Sinne, dass er die von der Letzteren gesetzte Kausalkette der Begriffe und Zwecke unterbricht. Die Dinge können nun unbegrifflich und unkausal wahrgenommen werden, jedoch bleibt formale Zweckmäfsigkeit als Idee bestehen, die jetzt aber von realen Zwecken befreit ist und nur noch ausgehöhlt-formal, dem Betrachter des Schönen in der Anschauung das Gefühl eines Gesamtzwecks gibt, das als Gefühl subjektiv ist und darum nicht mehr begrifflich benannt werden kann. In der vollends entwickelten ästhetischen Betrachtung fällt die reale Zweckmäßigkeit ohnehin weg und von der formalen wird zusätzlich noch der objektive Zweck abgezogen. Dieser Moment des Schönen lautet deshalb in der Kant'schen Formulierung:

"Schönheit ist die Form der Zweckmäßigkeit eines Gegenstandes, sofern sie ohne Vorstellung eines $Z$ wecks an ihm wahrgenommen wird. ${ }^{24}$

An dieser Stelle möchten wir auf die oben bereits angedeutete Schwierigkeit in Kants Ästhetik zurückkommen und fragen, was in diesem Spiel mit den Begriffen passiert. Kants Angaben dazu sind alles andere als eindeutig. Kant selbst sagt einmal, dass es keine Begriffsbildung im Geschmacksurteil gibt, ${ }^{25}$ dann aber auch, dass das Spiel, »ohne einen bestimmten Begriff vorauszusetzen «, ${ }^{26}$ entsteht und schließlich wiederum, dass es zustande kommt, weil

23 Ebd., S. AA $364 f$.

24 Ebd., S. AA 236.

25 Ebd., S. AA 211f., S. AA 219 und, wie hier zitiert, S. AA 229: »Schön ist das, was ohne Begriff [...] gefällt.«

26 Ebd., S. AA 217 [Hervorh. MG]. 
»die Einbildungskraft ohne Begriff schematisierk. ${ }^{27}$ Bei diesen unterschiedlichen Formulierungen verwundert es nicht, dass die Erkundung der Begriffe auch in der Kant-Forschung umstritten ist. Die Frage, zu der Kants so unterschiedlich definierte Begriffslosigkeit herausfordert, dass sie immer eine andere Schattierung bekommt und das Verhältnis der daraus entstehenden unterschiedlichen Momenten nicht verständlich wird, lautet: Wie kann der automatisch begriffsproduzierende Verstand ${ }^{28}$ sich auf die Einbildungskraft im "freien Spiel« beziehen, ohne Letztere durch Begriffe zu bestimmen? Das ästhetische Urteil schaltet ja den Verstand nicht einfach aus, sondern es kommt nur durch die Einbildungskraft zustande, sofern sich diese in einem Spiel zwischen Einbildungskraft und Verstand befindet. Dies aber bedeutet, dass der Verstand sozusagen immer noch rmitspielt und das Spiel Züge einer Formgebung trägt, die nicht allein der Einbildungskraft entspringen kann. Kant schreibt, dass Einbildungskraft und Verstand "zusammenstimmen $\aleph^{29}$ müssen und findet »nur da, wo Einbildungskraft in ihrer Freiheit den Verstand erweckt, und dieser ohne Begriffe die Einbildungskraft in ein regelmäßiges Spiel versetzt« die geforderte "Zusammenstimmung«. Die Einbildungskraft befindet sich im Spielzustand in »ihrer Freiheit«, jedoch ist es ein irgendwie geformtes, "regelmäßiges Spiel «. ${ }^{30}$ Anders gesagt: Hier ist eine uns bis jetzt unbekannte ,freie Gesetzmäßigkeit der Einbildungskraft am Werk, welche der Einbildungskraft einen autonomen Status verschafft und sie dadurch `von selbst ‘ gesetzmäßig werden lässt. Wie aber, so fragen wir weiter, kann die Einbildungskraft sich selbst ihre Gesetze geben, während doch der Verstand der alleinige Gesetzgeber ist; und wie, so könnte andersherum gefragt werden, kann der Verstand anwesend sein, ohne seine begriffliche Bestimmung ausüben zu können?

Die Standardlösung ${ }^{31}$ dieses Zirkels lautet, dass die Einbildungskraft hier keine Einheit mit einem bestimmten Begriff eingeht, sondern nur mit der allgemeinen Fähigkeit, Begriffe zu bilden. Die Anschauung wird also nicht so weit geformt, dass sie letztendlich mit einem Begriff verbunden ist, sondern wird inhaltsleer, im Sinne einer formalen Begriffsbildung bestimmt,

27 Ebd., S. AA 287 [Hervorh. MG].

28 Die Wahrnehmung von Objekten fällt immer unter das Gesetz der transzendentalen Analytik. D.h., wir brauchen immer einen empirischen Begriff, um die Synthese des Mannigfaltigen zu ermöglichen. Die Einbildungskraft synthetisiert das Mannigfaltige, aber nur durch die Verstandestätigkeit, kann das Ergebnis auch verstanden werden. Vgl. ebd., S. AA 217.

29 Ebd., S. AA 218.

30 Ebd., S. AA 296.

31 Vgl. Paul Guyer, Heinrich und Makkreel. 
indem sie lediglich mit dem allgemeinen Schematismus der Kategorien zusammengebracht wird. "Zweckmäßigkeit« könnte gemäß unserer Lesart auch 'Begriffsmäßigkeit‘ genannt werden, da kategorial schematisierte Anschauungen nur die Möglichkeit zur spezifischen Begriffsbildung enthalten, ohne diese jedoch zu realisieren. Diese formal-kategoriale Ordnung der Anschauung ist also sgröber als die begriffliche; die Kategorien spannen ein weitmaschiges Netz über die Wahrnehmung der Welt aus, das erst wenn die formale Begrifflichkeit mit empirischen Begriffen rangefüllt‘ wird, diese Weltsicht inhaltlich-begrifflich präzisieren. Ein anderer Ansatz zur Klärung der widersprüchlichen Begrifflichkeit im Geschmacksurteil kann in einem streng phänomenologischen Ansatz gefunden werden. ${ }^{32}$ Hier geht man davon aus, dass der Betrachter im ästhetischen Zustand in der reinen Wahrnehmung verweilt, also weder Kategorien noch Begriffe bildet und nur Farben, Formen und Töne erblickt, die nicht begrifflich geformt werden. Das ästhetische Urteil wird dieser Interpretation zufolge nur als ein Spiel akustischer oder visueller Differenzen vernommen - man denke hier an die Wahrnehmung einer Arabeske, die von Kant oft als ein Beispiel für ein reines Kunstwerk angeführt wird. Diese Leere in Kants Beispielen für uninteressierte Kunsterfahrung findet sich auch in seiner Vorliebe für Blumenmuster wieder und aufgrund des ausschließlich lieblich-ornamentalen Charakters seiner Bildlichkeit - \#Blumen, freie Zeichnungen, ohne Absicht ineinander verschlungene Züge, unter dem Namen eines Laubwerks « ${ }^{33}$ - ist seine reine Ästhetik deshalb von Spöttern auch schon mal als »Tapetenmuster-Ästhetik « ${ }^{34}$ bezeichnet worden. Und wenn Bildung das Bemühen beschreibt, Kunst mit Welterfahrung zusammenzubringen, dann sehen wir an dieser Stelle sehr gut, dass dies mit Kant nicht zu bewerkstelligen ist. In Goethes Bildungsroman Wilhelm Meisters Lehrjahre können wir gleich zu Beginn ein ähnliches Klagelied über die Schwierigkeit der Zusammenführung von Kunst und Welt sehen, wenn Wilhelm beim Anblick der Tapetenmuster im elterlichen Wohnzimmer in Wut gerät:

Als Wilhelm seine Mutter des andern Morgens begrüßte, eröffnete sie ihm, daß der Vater sehr verdrießlich sei und ihm den täglichen Besuch des Schauspiels nächstens untersagen werde. »Wenn ich gleich selbst«, fuhr sie fort, "manchmal gern ins Theater gehe, so möchte ich es doch oft verwünschen, da meine häusliche Ruhe durch

\footnotetext{
32 Vgl. David Bell, Crowford und Crowther.

33 Immanuel Kant, Kritik der Urteilskraft, S. AA 207.

34 Werner Strube, Interesselosigkeit. Zur Geschichte eines Begriffs in der Ästhetik, in: Archiv für Begriffsgeschichte 23 (1979), S. 170.
} 
deine unmäßige Leidenschaft zu diesem Vergnügen gestört wird. Der Vater wiederholt immer wozu es nur nütze sei? Wie man seine Zeit nur so verderben könne?« "Ich habe es auch schon von ihm hören müssen«, versetzte Wilhelm, "und habe $\mathrm{ihm}$ vielleicht zu hastig geantwortet; aber um Himmels willen, Mutter! ist denn alles unnütz, was uns nicht unmittelbar Geld in den Beutel bringt, was uns nicht den allernächsten Besitz verschafft? Hatten wir in dem alten Hause nicht Raum genug? und war es nötig, ein neues zu bauen? Verwendet der Vater nicht jährlich einen ansehnlichen Teil seines Handelsgewinnes zur Verschönerung der Zimmer? Diese seidenen Tapeten, diese englischen Mobilien, sind sie nicht auch unnütz? Könnten wir uns nicht mit geringeren begnügen? Wenigstens bekenne ich, daß mir diese gestreiften Wände, diese hundertmal wiederholten Blumen, Schnörkel, Körbchen und Figuren einen durchaus unangenehmen Eindruck machen. Sie kommen mir höchstens vor wie unser Theatervorhang. Aber wie anders ist's, vor diesem zu sitzen! Wenn man noch so lange warten muß, so weiß man doch, er wird in die Höhe gehen, und wir werden die mannigfaltigsten Gegenstände sehen, die uns unterhalten, aufklären und erheben ${ }^{35}$

Die erwünschte "häusliche Ruhe« der Mutter steht einem Unbehagen Wilhelms gegenüber, das von den Tapeten ausgeht und seinen Vorbehalt gegen die elterliche Langeweile zum Ausdruck bringt, welche sich nicht um das Erleben der Kunst bemüht, sondern sie in den immer gleichen privaten vier Wänden belässt. Der nutzlose Charakter der luxuriösen Einrichtung ist, in Bezug auf die Tapeten, für Wilhelm allenfalls ein ästhetisches Vorspiel, welches auf ein zukünftiges Versprechen in Form der Welt des Theaters verweist. Jedoch können die Wände nicht einfach verschwinden und wie ein Theatervorhang geöffnet werden und in eine andere Welt führen. Bedenkt man Wilhelms spätere Begeisterung für das Schauspiel und Shakespeare, dann wird leicht verständlich, wie gering die Bedeutung der "gestreiften Wände, dieser hundertmal wiederholten Blumen, Schnörkel, Körbchen und Figuren« im Verhältnis zu seiner kommenden Bildungsreise ist. Das Tapetenmuster verschnörkelt in Wilhelms Augen eine im elterlichen Haus existierende Leere, während sein eigenes Bildungsstreben »die mannigfaltigsten Gegenstände« zu sehen begehrt, »die uns unterhalten, aufklären und erheben «- all das, was das Schöne nach Kant nicht darf. Bildung bezieht sich vor allem auf die Sphäre der ranhängenden Schönheit‘, welche allerdings über vielfältigere Spielarten verfügt als in Kants Beschreibungen: ${ }^{36}$ Wilhelm möchte das Angenehme steigern und nicht in der freien Schönheit‘ verweilen. Er wünscht sich die ranhängende Schönheit in einer

35 Johann Wolfgang von Goethe, Wilhelm Meisters Lehrjahre, in: Ders., Goethes Werke, Hamburger Ausgabe, hg. von Erich Trunz, Hamburg 1950, S. 11f.

36 Vgl. Immanuel Kant, Kritik der Urteilskraft, S. AA 230. 
komplexeren Ausprägung. Gerade diese Schönheit ist es ja auch, die ihn aus der elterlichen Welt hinaus und in eine größere Welt drängt, in der er sich erhofft, seiner Leidenschaft als Schauspieler nachkommen zu können. Die freie Schönheit hätte ihn im Stadium des Betrachters gefangen gehalten, während das, was seiner Fantasie zufolge die Schönheit ranhängt‘, ihn dazu auffordert, diese aus der Schönheit resultierenden Ideen auch umzusetzen. Das Unnütze Wissen in Wilhelm Meister sind demzufolge seine Erfahrungen, die ihm bei diesem Weg von der reinen (und langweiligen) Schönheit wegführen und ihm anschließend in seinem neuen Leben als Schauspieler widerfahren und dadurch der zweckfreien ästhetischen Sphäre einen sozialen Kontext geben. Somit kann Literatur im Kontext der Fragestellung der vorliegenden Publikation als Spielwiese zunächst weltloser Ideen aufgefasst werden (Kants Ästhetik), die im Laufe ihrer Entwicklung eine konkrete Aufgabe erhalten (Schillers Bildungsidee) und sich dann im Bildungsroman im Schutze der Zweckscheinbarkeit - literarisch konkretisieren.

\section{Begriffsscheinbarkeit}

Die begrifflichen Abstraktionen des Zweckfreien bei Kant mit einer Bildlichkeit auf dem Niveau von Tapetenmustern appellieren an unsere Einbildungskraft. Wir dürfen uns von seiner angestrebten Präzision nicht irre machen und von seinen Bildern nicht langweilen lassen. Vielmehr gilt es, vor allem sein Begriffsgebäude als den Hintergrund zu verstehen, der unsere Fragestellung ausleuchtet und seine Bildlichkeit als einen Augenblick der konkreten Erfahrung, die es weiter zu beschreiben gilt. Anders gesagt: Bevor unsere Untersuchung an dieser Stelle anfängt, sich auf eine Kant-Exegese zu verengen, fassen wir lieber den bisherigen Horizont des Beobachtbaren zusammen, indem wir feststellen, dass die Frage nach den Begriffen im ästhetischen Urteil Kants abschließend nicht zu klären ist und an dieser Stelle im Sinne der Zweckscheinbarkeit eine Begriffsscheinbarkeit festgestellt werden kann. Dies bedeutet, dass es einerseits so zu sein scheint, als ob es Begriffe oder zumindest Formen der Begrifflichkeit gebe, aber andererseits kann die vermeintliche Begriffsbildung auch wieder über sich hinaus in das Unbegriffliche gehen. Es werden also Anstalten zur Begriffsbildung unternommen, ohne dass daraus letztendlich Begriffe entstehen müssen. Man erhält von Kant zahlreiche verfeinerte Begriffszuweisungen auf der Ebene des Schematismus und stellt in diesen Momenten Bewegungen im Hinblick auf 
Begriffsbildungen fest, welche den ästhetischen Zustand beschreiben sollen. Suchen wir die Klärung dieser Augenblicke jedoch ausschließlich innerhalb der scheinbaren Begrifflichkeit Kants, werden wir das Ästhetische weder befriedigend beschreiben noch ihm bedeutend näherkommen können. Ändern wir jedoch die Richtung unserer Untersuchung, indem wir die Scheinbarkeit als Hauptmoment des ästhetischen Zustands verstehen, kommen wir um die Hauptschwierigkeit der Kritik der Urteilskraft herum, die Zweckfreiheit durch penible und endlose Begriffsklärung verstehen zu müssen. Wir können uns diese Konzentration auf das Verweilen an einer spielerischen und sscheinbaren Stelle im Kant'schen System jedoch nur erlauben, weil wir - und das ist ein zentraler Punkt für den kommenden, sich hier aufbauenden Gedankengang - nicht wie Kant und viele seine Interpreten auf der Suche nach reinen ästhetischen Urteilen sind. ${ }^{37}$ Dies hat folgenden Grund: Das Kant'sche reine Geschmacksurteil - die begriffliche/unbegriffliche, d.h. die scheinbare "Zweckmäßigkeit ohne Zweck" - ist etwas Statisches und benennt einen kurzen Moment, den es eigentlich gar nicht gibt. In Wirklichkeit befinden wir uns nämlich immer in einem Prozess, der durch lauter unreine (Geschmacks-)Urteile konstituiert wird. Man urteilt in den seltensten Fällen entweder rein ästhetisch oder rein unästhetisch, sondern befindet sich meistens in einem Mischverhältnis dieser beiden Pole. Kants Ästhetik lässt eigentlich nur die Kontemplation in einer stillen Enklave zu, weil nur diese Orte genauso wie seine Theorie ohne Kontext verfasst sind. Seine Bilder und Tapetenmusterspiele, aber auch der Vogelgesang oder die Blumen, die Kant als Beispiele für seine Theorie dienen, tragen keine Züge ihrer Umgebung, in welcher sie dem Subjekt erscheinen. Sie sind alle rrein in dem Sinne, dass sie in keinen Zusammenhang eingebettet sind und entsprechen deshalb einer Richtung der Interpretation des ästhetischen Urteils, das die Schönheit als unbegrifflich ansieht, weil diese Beispiele hier ebenfalls bar jeder Kausalität - und das bedeutet bei Kant immer auch Begrifflichkeit - sind. Doch ebenso wie wir Probleme haben, diese Unbegrifflichkeit zu akzeptieren, so seltsam kommt uns auch die Kontextlosigkeit des ästhetischen Urteils vor. Die zweckgemäße Setzung eines Zwecks wird in der "Zweckmäßigkeit ohne Zweck"s schließlich bloß unterbrochen und mündet nicht in

37 Für eine Untersuchung des Kant'schen Schönheitsurteils, die nicht den Weg der analytischen Philosophie auf der Suche nach einer genauen Klärung des Zusammenspiels zwischen Verstand und Anschauung geht, sondern die angeschaute ästhetische Lust eindeutig vor der Erkenntnis ansetzt, weil diese mit dem Spiel beginnt und sich in der Erkenntnis vorsetzt vgl. Andrea Kern, Schöne Lust. Eine Theorie der ästhetischen Erfahrung nach Kant, Frankfurt a.M. 2000. 
den Prozess einer anderen Dimension der Weltwahrnehmung, wie es dem Auftrag der ästhetischen Bildung nach Schiller entspräche. Das »freie Spiel«, das Kant im ästhetischen Urteil am Werk sieht, wird von Schiller in ein größeres Projekt eingespannt, das sich durchaus um die Freiheit von ästhetischen Zuständen bemüht, jedoch in einem zweiten Schritt dieses weltlose Moment auch wieder überwinden möchte.

Unser Begriff der Zweckscheinbarkeit sieht das Ästhetische dagegen in einem sozialen Kontext. Kants ästhetische Erfahrung ist stets nur ein kurzer Moment, der aufgrund der strengen Reinheitsanforderung gleich auch schon wieder vorbei ist und mehr auf eine Idee als eine reale Erfahrung zurückgeht. Indem wir uns auf diese Erfahrung konzentrieren, kommen wir der Kraft des Zweckfreien - nämlich ihrer Zweckscheinbarkeit - näher und können die Kant'schen Verengungen ausweiten. 